\title{
How to Cultivate the Philosophical Thinking Ability of Science and Engineering Students in Classroom Teaching
}

\author{
Wenbing Wu, Rihua Liu, Yupeng Wu \\ Nanchang Normal University, Nanchang, China \\ Email: wwbysq@fjnu.edu.cn
}

How to cite this paper: Wu, W. B., Liu, R. H., \& Wu, Y. P. (2020). How to Cultivate the Philosophical Thinking Ability of Science and Engineering Students in Classroom Teaching. Creative Education, 11, 2207-2212. https://doi.org/10.4236/ce.2020.1111160

Received: August 22, 2020

Accepted: November 9, 2020

Published: November 12, 2020

Copyright (๑) 2020 by author(s) and Scientific Research Publishing Inc. This work is licensed under the Creative Commons Attribution International License (CC BY 4.0).

http://creativecommons.org/licenses/by/4.0/

\section{(c) (i) Open Access}

\begin{abstract}
In order to make the teaching of signal and system course more vivid, philosophical thinking is introduced in the process of classroom teaching by introducing the principle of universality and particularity of contradiction into the interpretation of Fourier series, the principle of unity and opposites of contradiction into the explanation of rectangular function and shock function, the principle of quantitative change and qualitative change into the theory of square wave function expansion, and the unity of finite and infinite opposition into the spectrum analysis of shock function. It can be closely combined. Teaching practice shows that this teaching method can effectively mobilize students' learning enthusiasm, expand students' thinking depth, and achieve better teaching effect.
\end{abstract}

\section{Keywords}

Signal and System, Philosophical Speculation, Thinking Depth, Teaching Method

\section{Introduction}

Philosophy is a discipline that conducts research on basic and universal issues, and is a theoretical system about world outlook and methodology. The world outlook is a general understanding of basic issues such as the nature of the world, the fundamental laws of development, and the fundamental relationship between human thinking and existence. Methodology is the way humans understand the world based on the world outlook. Philosophy is a method, not a set of propositions or theories. The study of philosophy is based on rational thinking, seeking to make hypotheses that have been reviewed or just pure anal- 
ogy. Philosophy is a thinking ability. Philosophy can start from an established concept system, concept or concept, and then integrate historical thinking technology to make one's thinking powerful, thereby further creating new knowledge.

With the rapid development and accelerated iteration of modern science and technology, cross-penetration between disciplines becomes more and more frequent, the boundaries between disciplines are becoming more and more blurred, and the boundaries between liberal arts and sciences are becoming more and more blurred. Contemporary university education in science and engineering is more inclined to cultivate talents with both advanced and deep professional knowledge in science and engineering, but also with profound humanistic literacy. Literature (Lin \& Shen, 2018; Xu, 2019; Li \& Wang, 2019; Chen, Zhang, \& Dong, 2015; Wang, Liu, \& Liang, 2017; Zeng, 2011) has made useful explorations in this area. At the same time, in the university education environment, classroom teaching runs through, which is the most direct and important way for students to receive education. In the classroom teaching of science and engineering college students, how to inspire students to carry out philosophical thinking about what they have learned, so as to enhance the depth and breadth of knowledge understanding, the author of the literature (Tong, Sun, \& Zheng, 2011; Ding, Chen, \& Ren, 2014; Yan-Yan M, 2019) has conducted a more in-depth study on this issue.

"Signal and System" is an important professional basic course for electrical majors. Its task is to study the basic theories and basic analysis methods of signals and linear time-invariant systems. It requires mastering the most basic signal transformation theory and the analysis methods of linear time-invariant systems. It is for learning follow-up courses and engaging in related fields. Engineering technology and scientific research work have laid a solid theoretical foundation. Through the study of this course, students will understand the function representation of signals and system analysis methods, and master the time domain analysis and frequency domain analysis methods of continuous-time systems and discrete-time systems.

Because there are many derivations and formulas in this course, many students feel boring and complicated when they study, and at the same time they feel that they have no connection with the feelings in real life, so some students are easy to lose interest in learning. In order to increase the students' interest in learning, the author of this article pays attention to integrating philosophical knowledge into the teaching when teaching this course. This will make the teaching of the course more lively and interesting, and at the same time make the students in the learning process be more proactive. This method of philosophical speculation enables students to further think about the ins and outs of these formulas while understanding the meaning of various formula symbols, and can also cultivate their creative thinking ability to a certain extent. Teaching practice shows that this method has achieved better teaching results. 


\section{Introduce the Principle of Universality and Particularity of Contradiction to Fourier Series}

French mathematician Fourier discovered that any periodic function can be represented by an infinite series composed of a sine function and a cosine function (the sine function and the cosine function are chosen as the basis functions because they are orthogonal), which is later called Fourier Series is a special trigonometric series. According to Euler's formula, trigonometric functions can be transformed into exponential form, which is also called Fourier series as an exponential series.

When explaining Fourier series, the author first introduces the concept of expansion from the most common one-dimensional, two-dimensional and three-dimensional spaces. As shown in Figure 1, in one-dimensional space, any point away from the source point has a value. This value can be regarded as the projection of this point in one-dimensional space.

Similarly, given two-dimensional rectangular coordinate system and three dimensional rectangular coordinate system: (Figure 2 \& Figure 3).

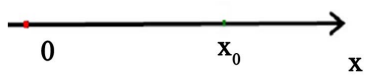

Figure 1. One-dimensional space.

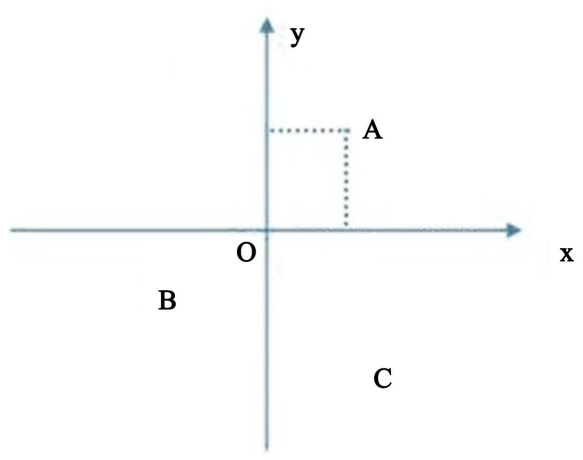

Figure 2. Two-dimensional space.

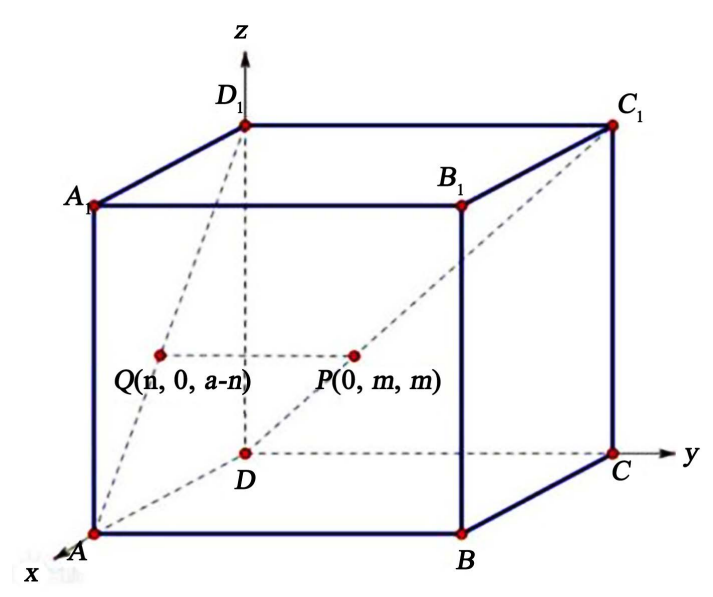

Figure 3. Three-dimensional space. 
Assuming that the coordinates of A are $(2,2)$, according to the vector representation, then $\mathrm{OA}=2 x+2 y, x, y$ represents the direction vector on the $x$ and $y$ axis.

Also given a three-dimensional rectangular coordinate system:

Assuming that the coordinates of $\mathrm{B} 1$ are $(3,3,3)$, according to the vector representation, then $\mathrm{OB}_{1}=3 \boldsymbol{x}+3 \boldsymbol{y}+3 \boldsymbol{z}, \boldsymbol{x}, \boldsymbol{y}, \boldsymbol{z}$ represent the direction vectors on the $x, y$, and $z$ axes respectively.

In other words, the space rectangular coordinate system decomposes any point in space to different coordinate axes. Obviously, the $x, y$, and $z$ axes are perpendicular to each other. In real space, no other axes can be found. They can be perpendicular to the $x, y$, and $z$ axes. Generally speaking, for any space the decomposition of one point will end everywhere. In order to elicit the concept of $\mathrm{n}$-dimensional space, according to the principle of universality and particularity in philosophy: Universality resides in particularity and is expressed through particularity. Without particularity, there is no universality. At the same time, particularity cannot be separated. Open universality. Based on this, it can be considered that 1, 2, 3 dimensional space is a special expression of universal $\mathrm{n}$-dimensional space, which leads to the concept of $\mathrm{n}$-dimensional space in which each axis is perpendicular to each other: (Figure 4)

Two functions orthogonal means that they satisfy: $\int_{t_{1}}^{t_{2}} f_{1}(t) f_{2}(t) \mathrm{d} t=0$.

Given function system $\{1, \cos x, \sin x, \cos 2 x, \sin 2 x, \cdots, \cos n x, \sin n x, \cdots\}$ It is called the trigonometric function system. Obviously $2 \pi$ is the period of all the above functions.

The orthogonality of the trigonometric function system on $[-\pi, \pi]$ means:

For any positive integer $n, m \quad(m \neq n)$, there is

$$
\begin{gathered}
\int_{-\pi}^{\pi} \cos n x \mathrm{~d} x=0, \int_{-\pi}^{\pi} \sin n x \mathrm{~d} x=0, \\
\int_{-\pi}^{\pi} \sin m x \cos n x \mathrm{~d} x=0, \int_{-\pi}^{\pi} \sin m x \sin n x \mathrm{~d} x=0, \int_{-\pi}^{\pi} \cos m x \cos n x \mathrm{~d} x=0, \\
\int_{-\pi}^{\pi} 1^{2} \mathrm{~d} x=2 \pi, \quad \int_{-\pi}^{\pi} \cos ^{2} n x \mathrm{~d} x=\int_{-\pi}^{\pi} \sin ^{2} n x \mathrm{~d} x=\pi
\end{gathered}
$$

Then $f(x)$ can be expanded to:

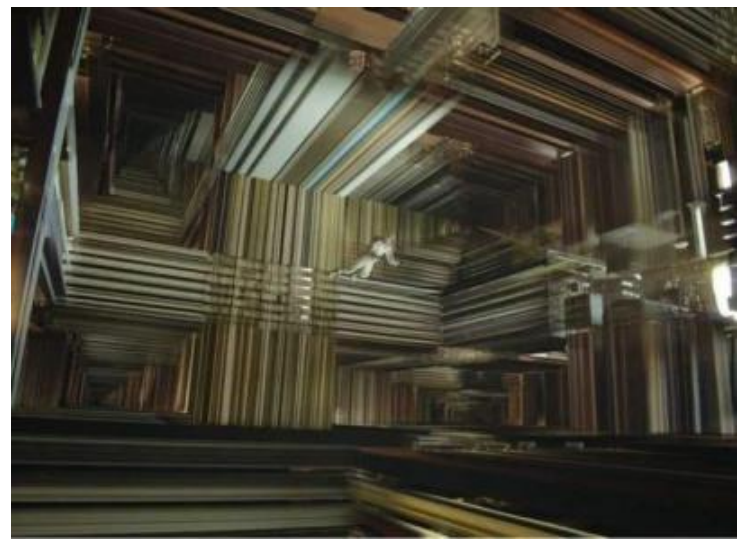

Figure 4. n-dimensional space. 


$$
\begin{gathered}
f(x)=\frac{a_{0}}{2}+\sum_{n=1}^{\infty}\left(a_{n} \cos n x+b_{n} \sin n x\right) \\
\left\{\begin{array}{l}
a_{n}=\frac{1}{\pi} \int_{-\pi}^{\pi} f(x) \cos n x \mathrm{~d} x, \quad n=0,1,2, \cdots, \\
b_{n}=\frac{1}{\pi} \int_{-\pi}^{\pi} f(x) \sin n x \mathrm{~d} x, \quad n=1,2, \cdots
\end{array}\right.
\end{gathered}
$$

Philosophical epistemology points out: Perceptual knowledge and rational knowledge are two different stages of knowledge in the process of knowledge. Perceptual knowledge is the lower stage of knowledge, characterized by directness and concreteness. Rational knowledge is the advanced stage of knowledge, including concepts, judgments, reasoning and other forms. The characteristic is its indirectness and abstraction. From the content point of view, the object of perceptual knowledge is the phenomenon of things, and the object of ideal knowledge is the essence of things. The low-dimensional space in this example can be regarded as a perceptual knowledge, and the n-dimensional space can be regarded as a rational knowledge. Therefore, the Fourier series expansion of the n-dimensional space can be derived from the simple coordinates of the low-dimensional space. It can also be considered as a process from perceptual knowledge to rational knowledge.

\section{Conclusion}

Philosophy has a guiding significance for the research of natural sciences. Therefore, by introducing philosophical viewpoints into a specific course, not only can the teaching effect be improved, but it can also guide students to think independently and enhance their innovative thinking ability to a certain extent. Of course, this method may not be very rigorous when linking a specific science formula or concept with a philosophical principle, but this does not prevent the achievement of the teaching purpose.

\section{Acknowledgements}

This paper is supported by Research Foundation of the Nanchang Normal University for Doctors (NSBSJJ2018014).

Key R \& D Projects of Jiangxi Provincial Department of Science and Technology: 20192BBHL80002, 20192BBEL50040.

\section{Conflicts of Interest}

The authors declare no conflicts of interest regarding the publication of this paper.

\section{References}

Chen, G. L., Zhang, L., \& Dong, R. S. (2015). University Computer Quality Education: Computational Culture, Computational Science and Computational Thinking. Chinese University Teaching, No. 6, 9-12. 
Ding, G. P., Chen, X. M., \& Ren, Y. Y. (2014). On Philosophical Thinking in Pathology Teaching. Journal of Nanjing Medical University (Social Science Edition), No. 4, 339-341.

Li, R. X., \& Wang, J. (2019). The Social Responsibility of the Core Literacy of High School Biology: Connotation, Advancement and Teaching Suggestions. Biology Bulletin, 54, 17-20.

Lin, L., \& Shen, S. S. (2018). Research on the Role of Design Thinking and Discipline Integration: The Cultivation Method of Core Competence in Basic Education. Audio-visual Education Research, 39, 12-18.

Tong, Y. P., Sun, H. B., \& Zheng, B. (2011). Applying Philosophy to Inspire Innovative Thinking in University Science Education. Higher Education Forum, No. 1, 42-44.

Wang, X. P., Liu, Y. L., \& Liang, Y. Y. (2017). Reform and Practice of Teaching Method, Learning Method and Examination Method of "Student-Centered". Chinese University Teaching, No. 6, 73-76.

$\mathrm{Xu}$, Y. C. (2019). Some Thoughts Based on the Cultivation of High School Biological Core Literacy. Encyclopedia Forum Electronic Magazine, No. 8, 508-509.

Yan-Yan M. (2019). Reflections on the Development of Philosophy and Social Sciences in Science and Engineering Colleges in the New Era. Journal of Hefei University (Natural Science Edition), 36, 10-14.

Zeng, Y. (2011). New Thoughts on Integrating and Transmitting Humanistic Spirit in Science Teaching: Taking Physical Chemistry Teaching as an Example. Higher Education Research (Chengdu), 28, 25-27. 